\title{
Prevention of cardiovascular disease: why do we neglect the most potent intervention?
}

\author{
Emmanuel Stamatakis, ${ }^{1,2}$ Richard Weiler ${ }^{3}$
}

Despite a large volume of evidence supporting its cardioprotective properties and its other numerous established health benefits, physical activity is not a serious prescription option for the primary prevention of cardiovascular disease. On the other hand, health services increasingly focus on pharmacological prevention without considering properly the long-term consequences of medication. Ethical and feasibility considerations suggest that evidence on the protective value of physical activity may need to be evaluated using criteria different from those applying to pharmacological trials. The collateral health benefits of physical activity prescription support its use as standard option in preventive health care.

Cardiovascular disease (CVD) is the main cause of death in developed countries and in the UK alone claims over 200000 lives per year. CVD is aetiologically complex and multifactorial. The majority of CVD risk factors are largely modifiable (eg, physical inactivity, smoking, poor diet, excessive alcohol consumption, stress), while others are less modifiable (eg, psychosocial factors) or unmodifiable (eg, genetic predispositions and age/sex). Complex and often poorly understood combinations of these risk factors result in disturbances to human physiology and biology, leading to the development of disease signs, such as obesity and type 2 diabetes, hypertension, dyslipidaemia and atherosclerosis. The consequence of these disturbances is expression of fatal or non-fatal CVD.

Physical inactivity and low cardiorespiratory fitness, its direct consequence, have been shown to be some of the largest aetiological contributors (along with smoking and diet) with hundreds of studies showing strong independent links with increased incidence of CVD and overall mortality. ${ }^{1}$ This is not reflected by current medical practice. Instead, the biological surrogate outcomes of poor lifestyles and stressful environments (eg,

\footnotetext{
'Department of Epidemiology, National Institute for Health Research Post-Doctoral Fellow, University College London, London, UK ${ }^{2}$ Department of Public Health, Senior Research Associate; National Institute for Health Research Post-Doctoral Fellow, University College London, London, UK ${ }^{3}$ Specialist Registrar in Sport and Exercise Medicine, London Deanery; General Practitioner, Hertfordshire, UK

Correspondence to Dr Emmanuel Stamatakis, Senior Research Associate, Department of Epidemiology and Public Health, University College London, 1-19 Torrington Place, London WC1E 6BT, UK; e.stamatakis@ucl.ac.uk
}

hypertension, obesity and raised blood cholesterol), are given "causal" factor status. With the exception of smoking, the actual behavioural causes of CVD are often called "predisposing" risk factors "...whose influence on CVD is due entirely to intensification of the causal factors (e.g. blood pressure, cholesterol, glucose)". ${ }^{2}$ Such logic has resulted in a disproportionate focus on pharmacology for CVD prevention, mainly lipid and blood pressurelowering medications. For example, in the case of statins, the evidence supporting their prescription for primary CVD prevention is highly controversial. ${ }^{3}$ Nevertheless, prescribing pressure comes from government health policy and in countries such as the United Kingdom general practitioners are incentivised to meet their contracts by prescribing to reduce cholesterol and blood pressure. While physical activity counselling is not a serious option (if an option at all), it is alarming that there are calls to even medicate whole populations regardless of baseline biological risk level. ${ }^{4}$

But what are the reasons for the apparent neglect of physical activity? First, although physical activity increases even in later life have been shown to decrease mortality risk as much as smoking cessation, ${ }^{5}$ there is limited randomised control trial (RCT) evidence showing that improvements in physical activity will lead to reduced mortality. In medical evidence hierarchy, RCTs are the only design that can support direct causal inferences but it is neither feasible nor ethical to instruct the control group of such a trial to be inactive for long periods of time. Thus, we may never be able to have the level of medical evidence required to make physical activity the first preventive option.
Second, there is a widespread belief among clinicians that people cannot change physical activity habits ${ }^{6}$ but we are not aware of any country where a substantial investment has been made to research which interventions might work in a primary care setting. For example, in the UK National Health Service there have only been a handful of physical activitypromoting initiatives in the past decade, all of which were of small scale and with minimal financial commitment. This is not justified as there is evidence showing that even brief consultations (3-10 minutes) or simple pedometer-based programmes delivered through health professionals can lead to substantial increases in patients' activity levels (by $\sim 30 \%)^{7}$ Conversely, adherence to medication is very low as only one in six patients takes their medication as prescribed $^{8}$ and approximately half of patients are sufficiently concordant in order to attain therapeutic objectives, yet medication is usually prescribed as a first option. The actual public health benefits of preventive CVD medication may be far lower than the, often questionable, ${ }^{3}$ RCT evidence upon which current practices are based. Worryingly, many general practitioners are not aware of the importance of physical activity ${ }^{9}$ and there are clear educational needs to be addressed. In addition, the average patient consultation lasts 9 minutes in the UK and the power to retain control over the consultation and prescribe a "pill for all ills" lies with the doctor. Unfortunately it is often far easier to issue a prescription to reduce blood pressure, cholesterol or body mass index targets than to fully address and follow up the multiple benefits and methods for improving a lifetime of risky lifestyle habits.

Third, the economies surrounding pharmaceutical companies have the power to fund and publicise research that supports the effectiveness of their products (and hence influence policy) and it is a well-known secret that pharmaceutical companies promote their agendas relentlessly by means of sponsorship and gifts among healthcare professionals (and hence influence practice). ${ }^{10}$ On the other hand, research on physical activity is sparingly funded.

Fourth, the frequently evidenced failure of the physical activity groups to meet the primary endpoint in trials reinforces views against physical activity. ${ }^{11}$ Again, it is unfair to judge a physical activity trial using the standard medical evidence criteria that invariably are concerned with dichotomous outcomes (success/failure). Because of the multiple benefits of 
increased physical activity and the different level of benefit any physical activity intervention can have, physical activity trials should be evaluated using multiple endpoints in a continuous fashion (very beneficial, beneficial, moderately beneficial, not beneficial, moderately harmful, etc). The dichotomous outcome research model is more appropriate for drug trials, with very limited or no collateral benefits and targeted action. All drugs have undesirable, known or unknown, side-effects that are often alleviated using other drugs that may in turn have more side-effects, making it nearly impossible to accurately track how medication affects quality of life in the long term. Side-effects are usually evaluated by indices of mortality and morbidity over a short period of time with little regard to how lifetime medication (like statins and $\beta$ blockers that are typically used for CVD prevention) may affect quality of life. On the other hand, the collateral benefits of regular physical activity are numerous and evidence suggesting new benefits is constantly emerging. Further, the degree of health benefits physical activity offers is likely to be greater than what the evidence suggests for reasons relating to how evidence has been produced: such evidence comes primarily from prospective studies with a single self-reported exposure assessment at baseline. True effect sizes are bound to be larger than what evidence suggest for at least two reasons: (a) the large measurement error associated with self-reporting weakens the strength of the associations between exposure and outcomes, and (b) unmeasured reductions in physical activity with age over the follow-up period that may subsequently dilute considerably effect sizes.
Finally, we speculate that relying merely on medication for CVD prevention may give a false sense of "I'm doing my bit for my health" and responsibility for health is transferred from the patient to the doctor via their concordance with medication. It has been shown that CVDfree individuals who are on CVD medication have lower adjusted odds to meeting the physical activity recommendations compared to those who are unmedicated. ${ }^{12}$

There is no doubt that CVD medication such as lipid lowering and blood pressure lowering can save lives but their establishment as a first-and-only primary preventive choice is scientifically unjustified and perhaps socially irresponsible. Developing and prescribing such drugs have taken large private and public investments and effort. It is high time the same investment is made in finding successful ways to facilitate and support active lifestyles for all. This not only will minimise the burden of cardiovascular and other chronic disease but it will also help people to live happier and fuller lives. As Hippocrates said, "if we could give every individual the right amount of nourishment and exercise, not too little and not too much, we would have found the safest way to health" and "walking is the man's best medicine". Two thousand four hundred years later it is not too late to put these principles into practice.

Acknowledgements $E S$ is funded by the National Institute for Health Research.

\section{Competing interests None.}

Provenance and peer review Not commissioned; externally peer reviewed.

Accepted 20 October 2009

Published Online First 5 November 2009
Heart 2010;96:261-262.

doi:10.1136/hrt.2009.186593

\section{REFERENCES}

1. Warburton DER, Katzmarzyk PT, Rhodes RE, et al. Evidence-informed physical activity guidelines fair Canadian adults. Appl Physiol Nutr Metab 2007;32:S16-68.

2. Grundy SM, Scott M. Primary prevention of coronary heart disease. Circulation 1999:100:988-98.

3. Wright JA. Are lipid-lowering guidelines evidencebased? Lancet 2007;369:168-9.

4. Law MR, Morris JK, Wald NJ. Use of blood pressure lowering drugs in the prevention of cardiovascular disease: meta-analysis of 147 randomised trials in the context of expectations from prospective epidemiological studies. BMJ 2009;338: b1665.

5. Byberg L, Melhus H, Gedeborg R, et al. Total mortality after changes in leisure time physical activity in 50 year old men: 35 year follow-up of population based cohort. $\mathrm{Br} J$ Sports Med 2009;43:482.

6. Ornish D. Statins and the soul of medicine. Am J Cardiol 2002;89:1286-90.

7. Marcus BH, Williams DM, Dubbert PM, et al. Physical activity intervention studies: what we know and what we need to know: a scientific statement from the American Heart Association Council on Nutrition, Physical Activity, and Metabolism (Subcommittee on Physical Activity); Council on Cardiovascular Disease in the Young; and the Interdisciplinary Working Group on Quality of Care and Outcomes Research. Circulation 2006;114:2739-52.

8. Oxford handbook of general practice. Compliance. Oxford: Oxford Handbooks, 94-95.

9. Gupta K, Fan L. Doctors: fighting fit or couch potatoes? Br J Sports Med 2009;43:153-4.

10. Greenland $\mathbf{P}$. Time for the medical profession to act: new policies needed now on interactions between pharmaceutical companies and physicians. Arch Intern Med 2009;169:829-31.

11. Mitka M. Lifestyle modification and heart disease. Researchers not deterred by trials show no benefit. JAMA 2009;301:150-1.

12. Stamatakis $\mathbf{E}$, Hamer $M$, Primatesta $P$. Cardiovascular medication, physical activity and mortality: cross-sectional population study with ongoing mortality follow-up. Heart 2009;95:448-53. 